FTUV-08-1507

\title{
Tau spin correlations and the anomalous magnetic moment
}

\author{
J. Bernabéu ${ }^{\text {a }}$, G. A. González-Sprinberg ${ }^{\text {b }}$ J. Vidal $^{\text {a }}$ \\ a Departament de Física Teòrica Universitat de València, E-46100 \\ Burjassot, València, Spain \\ and \\ IFIC, Centre Mixt Universitat de València-CSIC, València, Spain \\ ${ }^{\mathrm{b}}$ Instituto de Física, Facultad de Ciencias, Universidad de la República, Iguá 4225, \\ 11400 Montevideo, Uruguay
}

\begin{abstract}
We show that the precise determination of the Tau magnetic properties is possible in the next generation accelerators, specially at B/Flavour factories. We define spin correlation observables suitable to extract the real part of the magnetic form factor that, for the first time, will allow to test the standard model-QED predictions. In particular, the predicted QED-dependence with both the momentum transfer and the lepton mass can be precisely measured. Until now, the most stringent bounds on the $\tau$ magnetic moment $a_{\tau}$ come from LEP data with strong assumptions on the physics involved on the observed process. In this paper, we find three different combinations of spin correlations of the outgoing Taus that disentangle the magnetic moment form factor of the Tau lepton in the electromagnetic vertex. These combinations of asymmetries also get rid off the contributions coming from Z-mediating amplitudes to the defined correlations. Using unpolarized electron beams and an integrated luminosity of $15 \times 10^{18} b^{-1}$, the sensitivity to the $\tau$ magnetic moment form factor is of the order $10^{-6}$. This sensitivity is two orders of magnitude better than the present existing high- or low-energy bounds on the magnetic moment and would allow its actual measurement with the precision of a few per cent.
\end{abstract}

\section{Introduction}

The fact that the $\tau$ magnetic properties cannot be investigated in the way as done for a long lived particle, where the interaction with an external magnetic field can be directly measured, makes more subtle and difficult its determination. As stated in the PDG, the experimental determination of the $\tau$ magnetic moment, by the DELPHI Collaboration [1], was done using LEP2 data for 
the $e^{+} e^{-} \rightarrow e^{+} e^{-} \tau^{+} \tau^{-}$total cross-section, assuming that any deviation from the tree level SM prediction was exclusively due to the magnetic anomaly [2], $a_{\tau}=(g-2) / 2$. This indirect measurement bounds the $\tau$ magnetic moment to the values [3]:

$$
-0.052<a_{\tau}<0.013(95 \% \text { C.L. })
$$

In fact, with such assumptions, in some of the quoted experiments the bound that is effectively set is the contribution of new physics to the magnetic moment. This is the point of view adopted in [4], where the most stringent modelindependent limit for the magnetic properties is obtained:

$$
-0.007<a_{\tau}^{\text {NewPhys. }}<0.005(95 \% \text { C.L. })
$$

The bound given in Eq.(11) is well above the SM prediction [5]

$$
a_{\tau}^{S M}=1177.21(5) \times 10^{-6}
$$

where higher-order QED, hadronic and weak corrections are included and contribute only to the last figures of this value. In fact, present experimental bounds on the $\tau$ lepton magnetic moment anomaly are still more than one order of magnitude bigger than the result obtained by Schwinger [6] at oneloop in QED:

$$
a_{e}=a_{\tau}=\frac{\alpha}{2 \pi} \simeq 0.00116
$$

Super B/Flavour factories will produce in the future about $10^{12}$ Tau pairs [7], so that high precision measurements of the poorly know properties of the Tau lepton will be possible. In this paper we present a set of new observables appropriate to measure the magnetic moment form factor of the $\tau$ by analyzing the angular distribution of their decay products in $e^{+} e^{-}$collisions. For unpolarized electron beams, the imaginary part of the magnetic moment can be observed by measuring the normal polarization of a single Tau [8], whereas we show here that only spin correlations of the outgoing Taus are sensible to the real part of the magnetic moment. For polarized electron beams, we have shown in Ref. [8] that the real part of the magnetic moment can be observed by measuring the transverse and longitudinal polarization of a single Tau. We find that a sensitivity of the order of $10^{-6}$ can be achieved using high statistics facilities for Tau pair production on the top of the $\Upsilon$ resonance. Comparable sensitivities were found in Ref. 8] by using polarized electron beams and observables built on the polarization analysis of a single Tau. 


\section{Magnetic moment form factor}

The most general Lorentz invariant structure describing the interaction of a vector boson $V$ with two on-shell fermions $f \bar{f}$ can be written in terms of the conserved current $J^{\mu}$ as:

$$
\begin{aligned}
& \left\langle f\left(p_{-}\right) \bar{f}\left(p_{+}\right)\left|J^{\mu}(0)\right| 0\right\rangle= \\
& \quad e \bar{u}\left(p_{-}\right)\left[\gamma^{\mu} F_{1}+\frac{\left(i F_{2}+F_{3} \gamma_{5}\right)}{2 m_{f}} \sigma^{\mu \nu} q_{\nu}+\left(q^{2} \gamma^{\mu}-q^{\mu} \not 1\right) \gamma_{5} F_{A}\right] v\left(p_{+}\right)
\end{aligned}
$$

where $F_{1}$ is the Dirac form factor $\left(F_{1}(0)=1\right), F_{A}$ is the anapole moment, whereas $F_{2}$ and $F_{3}$ parametrize the magnetic and electric dipole moments, respectively.

The determination of the $C P$ - violating electric dipole moment, in Super B/Flavour factories, has been studied in detail in [9,10] with similar techniques to the ones presented here. The $P$-odd, $T$-even anapole moment differs from zero due to weak virtual corrections so that its contribution will be suppressed by factors of $q^{2} / M_{Z}^{2}$ compared to the leading QED corrections considered in this paper. The induced magnetic moment form factor $F_{2}$ is a chirality flip

observable in the vector current and its determination is the subject of this paper.

Strictly speaking, the magnetic moment anomaly

$$
a_{f} \equiv F_{2}\left(q^{2}=0\right)
$$

is defined with all three fields entering into the interaction vertex on their mass-shell. In Super B factories the squared center-of-mass energy $s=q^{2}$ is of the order $(10 \mathrm{GeV})^{2}$ and, therefore, $F_{2}\left(q^{2}\right)$ is no longer the magnetic anomaly. Note that if the evolution scale is well above $q^{2}$, as it is the case for new physics, then $F_{2}\left(q^{2}\right) \simeq F_{2}\left(q^{2}=0\right.$ ) (see Ref. [4) but in the case of interest, for QED, the evolution scale of the form factor is $m_{\tau}^{2}<q^{2}$ and the actual value of the form factor for B-Factories is quite different from the magnetic moment anomaly. The direct computation of the magnetic part of the standard one-loop QED vertex yields

$$
F_{2}(s)=\left(\frac{\alpha}{2 \pi}\right) \frac{2 m_{\tau}^{2}}{s} \frac{1}{\beta}\left(\log \frac{1+\beta}{1-\beta}-i \pi\right), \quad \text { for } \quad q^{2}=s>4 m_{\tau}^{2}
$$

where $\alpha$ is the fine structure constant and $\beta=\left(1-4 m_{\tau}^{2} / s\right)^{1 / 2}$ is the velocity of the $\tau$. 
The $F_{2}(s)$ form factor is gauge invariant, despite being an off-shell amplitude. This is so due to the specific gauge-cancellation mechanism that operates only in an abelian theory such as QED: the direct box cancels against the crossed one, the vector proper vertex against self-energy fermions graphs so the magnetic moment form factor, proportional to $\sigma_{\mu \nu} q^{\nu}$, must be separately gauge-independent.

In our case, for $q^{2} \sim M_{\Upsilon}^{2} \sim(10 \mathrm{GeV})^{2}$,

$$
F_{2}\left(M_{\Upsilon}^{2}\right)=(2.65-2.45 i) \times 10^{-4}
$$

This equation shows that, at this energy, the real and imaginary parts are of the same order and, due to the scale and flavour dependence, about 1/4 of the on-shell magnetic moment. This fact gives us the opportunity to see the behaviour of the form factor with the momentum $q^{2}$, for a pure abelian theory as QED, together with its strong dependence on the flavour (mass) of the fermion. Note that for other lighter fermions (as the electron) the magnetic moment form factor is vanishing small at the $M_{\Upsilon \text {-energy. }}$

For the extraction of $F_{2}$, in order to eliminate contamination from the box diagrams to the measured amplitude, we perform our analysis on top of the Upsilon resonance, so that kinematics makes the non-resonant box contributions negligible.

\section{$3 e^{+} e^{-} \longrightarrow \tau^{+} \tau^{-}$at Super B Factories.}

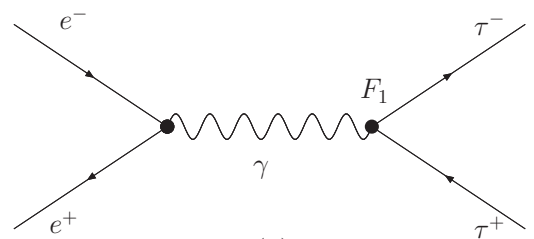

(a)

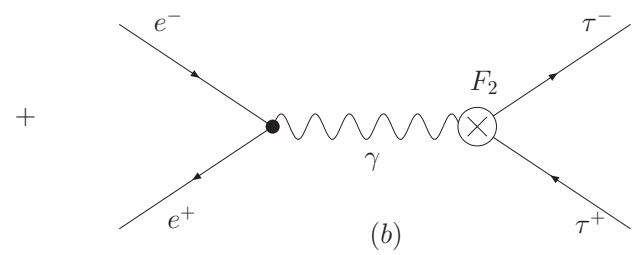

(b)

Fig. 1. Diagrams: (a) direct $\gamma$ exchange, (b) $F_{2}$ in $\gamma$ exchange.

In this section we first consider the $\tau$-pair production in $e^{+} e^{-}$collisions through direct $\gamma$ exchange (diagrams (a) and (b) in Fig. 1). Next, we will show that the basic results of this section still hold for resonant $\Upsilon$ production.

The differential cross section for $\tau$ pair production, with spin $\vec{s}_{+}, \vec{s}_{-}$, is:

$$
\frac{d \sigma\left(\vec{s}_{+}, \vec{s}_{-}\right)}{d \Omega_{\tau^{-}}}=\frac{d \sigma^{0}\left(\vec{s}_{+}, \vec{s}_{-}\right)}{d \Omega_{\tau^{-}}}+\frac{d \sigma^{S}\left(\vec{s}_{+}, \vec{s}_{-}\right)}{d \Omega_{\tau^{-}}}+\frac{d \sigma^{S S}\left(\vec{s}_{+}, \vec{s}_{-}\right)}{d \Omega_{\tau^{-}}}
$$


The first term of Eq.(9) represents the $\tau$ spin-independent differential cross section

$$
\frac{d \sigma^{0}\left(\vec{s}_{+}, \vec{s}_{-}\right)}{d \cos \theta_{\tau^{-}}}=\frac{\pi \alpha^{2}}{8 s} \beta\left[\left(2-\beta^{2} \sin ^{2} \theta_{\tau^{-}}\right)\left|F_{1}(s)\right|^{2}+4 \operatorname{Re}\left\{F_{1}(s) F_{2}(s)^{*}\right\}\right]
$$

where $\alpha$ is the fine structure constant, the squared center of mass energy $s=q^{2}$ is also the square of the 4-momentum carried by the photon, and $\gamma=$ $\sqrt{s} /\left(2 m_{\tau}\right), \beta=\sqrt{1-1 / \gamma^{2}}$, are the dilation factor and $\tau$ velocity, respectively. Note that, at one loop, we have the identity

$$
\operatorname{Re}\left\{F_{1}(s) F_{2}(s)^{*}\right\}=\operatorname{Re}\left\{F_{2}(s)\right\} .
$$

The $\theta_{\tau^{-}}$angle is determined in the center-of-mass $(\mathrm{CM})$ frame by the outgoing $\tau^{-}$and the incoming $e^{-}$momenta. In $\mathrm{B} /$ Flavour factories, a precise measurement of the $\theta_{\tau^{-}}$angle requires that the $\tau$ production plane and direction of flight are fully reconstructed. In Ref. [11] it is shown that this can be achieved if both $\tau$ 's decay semileptonically.

The second term $\frac{d \sigma^{S}}{d \Omega_{\tau^{-}}}$involves linear terms in the spin of the taus,

$$
\frac{d \sigma^{S}\left(\vec{s}_{+}, \vec{s}_{-}\right)}{d \cos _{\tau^{-}}}=\frac{\pi \alpha^{2}}{4 s} \beta\left(s_{-}+s_{+}\right)_{y} Y_{+}
$$

where $Y_{+}$, up to the considered order, is given by

$$
Y_{+}=\gamma \beta^{2}\left(\cos \theta_{\tau^{-}} \sin \theta_{\tau^{-}}\right) \operatorname{Im}\left\{F_{2}(s)\right\}
$$

Eq.(13) shows that the contribution of the chirality flipping $F_{2}$ to the normal polarization is enhanced by a factor of $\gamma$ with respect to other possible nonchirality flipping contributions. This observable is a $P$-even, $T$-odd quantity, as corresponds to the interference $\operatorname{Im}\left\{F_{1}(s) F_{2}(s)^{*}\right\}$.

We work in the center of mass (CM) frame of reference, and the orientation of the coordinate system is the same as in Ref. [9]. The $\boldsymbol{s}_{ \pm}$vectors are the $\tau^{ \pm}$ spin vectors in the $\tau^{ \pm}$rest system, $s_{ \pm}=\left(0, s_{ \pm}^{x}, s_{ \pm}^{y}, s_{ \pm}^{z}\right)$. Polarizations along the directions $x, y, z$ correspond to what is called transverse, normal, and longitudinal polarizations, respectively.

As shown in Eq.(13), the normal polarization of the outgoing Tau has sensitivity to the imaginary part of the magnetic moment form factor. In absence of $P$-violation, the other components of a single $\tau$ polarization cannot appear. 
The polarization of the final fermion $\left(\tau^{ \pm}\right)$can be studied by looking at the angular distribution of its decay products [8].

The last term of Eq.(10) is proportional to the product of the spins of both $\tau$ 's:

$$
\begin{array}{r}
\frac{d \sigma^{S S}\left(\vec{s}_{+}, \vec{s}_{-}\right)}{d \cos \theta_{\tau^{-}}}=\frac{\pi \alpha^{2}}{8 s} \beta\left(s_{+}^{x} s_{-}^{x} C_{x x}+s_{+}^{y} s_{-}^{y} C_{y y}+s_{+}^{z} s_{-}^{z} C_{z z}\right. \\
\left.+\left(s_{+}^{x} s_{-}^{z}+s_{+}^{z} s_{-}^{x}\right) C_{x z}^{+}\right)
\end{array}
$$

where

$$
\begin{aligned}
& C_{x x}=\left(\left(2-\beta^{2}\right)\left|F_{1}\right|^{2}+4 \operatorname{Re}\left\{F_{2}\right\}\right) \sin ^{2} \theta_{\tau^{-}} \\
& C_{y y}=-\left|F_{1}\right|^{2} \beta^{2} \sin ^{2} \theta_{\tau^{-}} \\
& C_{z z}=\left|F_{1}\right|^{2}\left(2 \cos ^{2} \theta_{\tau^{-}}+\beta^{2} \sin ^{2} \theta_{\tau^{-}}\right)+4 \operatorname{Re}\left\{F_{2}\right\} \cos ^{2} \theta_{\tau^{-}} \\
& C_{x z}^{+}=\left(\left|F_{1}\right|^{2}+\gamma^{2}\left(2-\beta^{2}\right) \operatorname{Re}\left\{F_{2}\right\}\right) \frac{1}{\gamma} \sin \left(2 \theta_{\tau^{-}}\right)
\end{aligned}
$$

Equation (14) shows that, as expected in absence of any source of $P$-violation, only $P$-even correlations can contribute to the spin-spin differential cross section. All the terms are also $T$-even, so only $\operatorname{Re}\left\{F_{2}\right\}$ appears. The $C_{x y}$ and $C_{y z}$ correlation terms, being $P$-odd, cannot appear in the given cross section.

The $C_{x z}$ term is the only chirality flipping correlation of the process and then it has the $F_{2}$ contribution enhanced by the dilation factor $\gamma$, whereas the $\left|F_{1}\right|^{2}$ form factor, which is chirality conserving, is suppressed by the Tau mass $(1 / \gamma$ factor).

Following Ref. [12, the differential cross-section for the process

$$
e^{+} e^{-} \rightarrow h^{+} \bar{\nu}_{\tau} h^{-} \nu_{\tau}
$$

can now be obtained by convoluting the previous $d \sigma\left(e^{+} e^{-\stackrel{\gamma}{\rightarrow}} \tau^{+}\left(\vec{s}_{+}\right) \tau^{-}\left(\vec{s}_{-}\right)\right)$, differential cross section, with the Tau decay branching ratios, to get

$$
\begin{aligned}
d \sigma\left(e^{+} e^{-\tau^{+} \tau^{-}} h^{+} \bar{\nu}_{\tau} h^{-} \nu_{\tau}\right)= & 4 d \sigma\left(\vec{n}_{+}^{*}, \vec{n}_{-}^{*}\right) \frac{d \Omega_{h^{+}}}{4 \pi} \frac{d \Omega_{h^{-}}}{4 \pi} \\
& \times B r\left(\tau^{-} \rightarrow h^{-} \nu_{\tau}\right) \operatorname{Br}\left(\tau^{+} \rightarrow h^{+} \bar{\nu}_{\tau}\right)
\end{aligned}
$$

where the spins $\vec{s}_{ \pm}$in the differential cross-section (9) have been substituted by

$$
\vec{n}_{ \pm}^{*}=\mp \alpha_{ \pm} \frac{\vec{q}_{ \pm}^{*}}{\left|\vec{q}_{ \pm}^{*}\right|}=\mp \alpha_{ \pm}\left(\sin \theta_{ \pm}^{*} \cos \phi_{ \pm}, \sin \theta_{ \pm}^{*} \sin \phi_{ \pm}, \cos \theta_{ \pm}^{*}\right)
$$


which are proportional to the momenta of the hadrons $\left(h^{ \pm}\right)$with moduli fixed to the polarization analyzer

$$
\alpha_{h} \equiv \frac{m_{\tau}^{2}-2 m_{h}^{2}}{m_{\tau}^{2}+m_{h}^{2}} .
$$

The $\phi_{ \pm}$and $\theta_{ \pm}^{*}$ angles are the azimuthal and polar angles of the produced hadrons $h^{ \pm}\left(\hat{q}_{ \pm}^{*}\right)$ in the $\tau^{ \pm}$rest frame (the ${ }^{*}$ means that the quantity is given in the $\tau$ rest frame). From Eq.(19), integrating over $\Omega_{ \pm}$and $\phi_{\tau^{-}}$angles, the spin-independent cross-section is

$$
\begin{aligned}
\frac{d \sigma}{d\left(\cos \theta_{\tau^{-}}\right)} & \left(e^{+} e^{-} \stackrel{\tau^{+} \tau^{-}}{\rightarrow} h^{+} \bar{\nu}_{\tau} h^{-} \nu_{\tau}\right)=\frac{\pi \alpha^{2}}{2 s} \beta\left[\left(2-\beta^{2} \sin ^{2} \theta_{\tau^{-}}\right)\left|F_{1}(s)\right|^{2}\right. \\
& \left.+4 \operatorname{Re}\left\{F_{2}(s)\right\}\right] \operatorname{Br}\left(\tau^{-} \rightarrow h^{-} \nu_{\tau}\right) \operatorname{Br}\left(\tau^{+} \rightarrow h^{+} \bar{\nu}_{\tau}\right)
\end{aligned}
$$

By integrating over $\theta_{\tau^{-}}$, Eq.(22) will provide, to leading order in $\alpha$ (that means taking $F_{1}=1$, and $F_{2}=0$ ), the normalization for all the asymmetries considered in this paper.

This equation shows that, a priori, the real part of $F_{2}$ could be measured from the cross section. However, this determination would be directly affected by the precision in the reconstruction of the Tau direction, so that problems with dilution, kinematic reconstruction and/or efficiency over $\cos \theta_{\tau^{-}}$, make difficult the extraction of $F_{2}$ from the cross-section.

The imaginary part of $F_{2}$ is a $T$-odd, $C$ - and $P$-even quantity; therefore, a suitable observable to look for its determination will be the normal (to the scattering plane) polarization of the outgoing $\tau$, as discussed after Eq.(13). The polarization analyzer has been studied in detail in Ref. [8] and the results are shown in Table 1.

In absence of electron polarization, only spin-spin correlations of the Tau decay products allow the determination of the real part of the $F_{2}$ magnetic moment form factor. This will be the object of the following sections.

\section{Tau spin-spin correlations}

In this section we study several spin correlation observables proportional to the magnetic form factor $F_{2}$ that could be measured at B-Factories. We will sum the events for all angles as possible in the angular distribution in such a way as to maintain the information on the magnetic form factor. In subsection 
4.1 we first consider the case for direct Tau pair production, in subsection 4.2 we study the resonant Tau pair production observables and in subsection 4.3 we compute the contributions from $Z-\gamma$ interference.

\subsection{Direct production}

From Eq.(14), it is straightforward to see that the real part of the $F_{2}$ form factor can only be measured by appropriate linear combinations of the different spin correlation terms shown there, i.e, Transverse-Transverse $(T T \equiv$ $x x)$, Normal-Normal $(N N \equiv y y)$, Longitudinal-Longitudinal $(L L \equiv z z)$ and Longitudinal-Transverse $(L T \equiv z x)$ to the scattering plane.

\subsubsection{With Tau-direction symmetric integration}

Integrating out $\cos \theta_{\tau^{-}}$in Eq.(14) one gets that the information carried by the LT correlation is erased, so that only $T T, N N$ and $Z Z$ correlations survive

$$
\begin{aligned}
d^{4} \sigma^{S S}=\frac{2 \pi \alpha^{2} \beta}{3 s}\left[\left(s_{+}^{x} s_{-}^{x}\right) \mathcal{X} \mathcal{X}\right. & \left.+\left(s_{+}^{y} s_{-}^{y}\right) \mathcal{Y} \mathcal{Y}+\left(s_{+}^{z} s_{-}^{z}\right) \mathcal{Z Z}\right] \\
\times & \frac{d \Omega_{h^{+}}}{4 \pi} \frac{d \Omega_{h^{-}}}{4 \pi} B r_{+} B r_{-}
\end{aligned}
$$

being $B r_{ \pm}$the decay branching ratios of Taus to charged hadrons plus neutrinos, $B r_{-}=\operatorname{Br}\left(\tau^{-} \rightarrow h^{-} \nu_{\tau}\right), B r_{+}=\operatorname{Br}\left(\tau^{+} \rightarrow h^{+} \bar{\nu}_{\tau}\right)$, and

$$
\begin{aligned}
& \mathcal{X X}=\left(2-\beta^{2}\right)\left|F_{1}\right|^{2}+4 \operatorname{Re}\left\{F_{2}\right\} \\
& \mathcal{Y} \mathcal{Y}=-\left|F_{1}\right|^{2} \beta^{2} \\
& \mathcal{Z} \mathcal{Z}=\left(1+\beta^{2}\right)\left|F_{1}\right|^{2}+2 \operatorname{Re}\left\{F_{2}\right\}
\end{aligned}
$$

For disentangling the relevant $F_{2}$ form factor from the spin-spin correlations one may now integrate appropriately the angular variables $\left(\Omega_{ \pm}\right)$of the final hadrons. For each correlation, we show the way how this integration has to be done: the $T T$ and $N N$ terms lead to azimuthal asymmetries of the decay products, whereas the $L L$ term leads to a polar asymmetry.

- For the TT correlation, we integrate in Eq.(23) the polar $\theta_{ \pm}^{*}$ angles to get

$$
d^{2} \sigma_{T T}=\frac{\pi \alpha^{2} \beta}{96 s}\left[-\left(\alpha_{-} \alpha_{+}\right)\right](\mathcal{X} \mathcal{X})\left(\cos \phi_{-} \cos \phi_{+}\right) d \phi_{+} d \phi_{-} B r_{+} B r_{-}
$$

and then perform an asymmetric integration over the azimuthal angles $\phi_{ \pm}$. In this way we define the TT-asymmetry: 


$$
\begin{aligned}
A_{T T} & \equiv \frac{-\left(\alpha_{-} \alpha_{+}\right)}{\sigma}\left(\int_{-\pi / 2}^{\pi / 2} d \phi_{-}-\int_{\pi / 2}^{3 \pi / 2} d \phi_{-}\right)\left(\int_{-\pi / 2}^{\pi / 2} d \phi_{+}-\int_{\pi / 2}^{3 \pi / 2} d \phi_{+}\right) d^{2} \sigma_{T T} \\
& =-\frac{\pi \alpha^{2} \beta}{6 s} \frac{\alpha_{-} \alpha_{+}}{\sigma}\left[\left(2-\beta^{2}\right)\left|F_{1}\right|^{2}+4 \operatorname{Re}\left\{F_{2}\right\}\right] B r_{+} B r_{-}
\end{aligned}
$$

with $\sigma$ (total cross section) given by the integration of Eq.(22).

- For the $N N$ term, we follow a similar procedure, integrating out the $\theta_{ \pm}^{*}$ polar angles to get

$$
\begin{aligned}
d^{2} \sigma_{N N}=\frac{\pi \alpha^{2} \beta}{96 s}\left[-\left(\alpha_{-} \alpha_{+}\right)\right] & (\mathcal{Y Y})\left(\sin \phi_{-} \sin \phi_{+}\right) d \phi_{+} d \phi_{-} \\
& \times B r_{+} B r_{-}
\end{aligned}
$$

and then we integrate asymmetrically the azimuthal $\phi_{ \pm}$angles, in order to define to obtain the $N N$-asymmetry:

$$
\begin{aligned}
A_{N N} & \equiv-\frac{\alpha_{-} \alpha_{+}}{\sigma}\left(\int_{0}^{\pi} d \phi_{-}-\int_{\pi}^{2 \pi} d \phi_{-}\right)\left(\int_{0}^{\pi} d \phi_{+}-\int_{\pi}^{2 \pi} d \phi_{+}\right) d^{2} \sigma_{N N} \\
& =\frac{\pi \alpha^{2} \beta}{6 s} \frac{\left(\alpha_{-} \alpha_{+}\right)}{\sigma} \beta^{2}\left|F_{1}\right|^{2} B r_{+} B r_{-}
\end{aligned}
$$

- Finally, for the $L L$ correlation we must first integrate the azimuthal $\phi_{ \pm}$ angles to get

$$
\begin{aligned}
d^{2} \sigma_{L L}=\frac{\pi \alpha^{2} \beta}{6 s}\left[-\left(\alpha_{-} \alpha_{+}\right)\right](\mathcal{Z Z}) & \left(\cos \theta_{-}^{*} \cos \theta_{+}^{*}\right) d\left(\cos \theta_{+}^{*}\right) d\left(\cos \theta_{-}^{*}\right) \\
\times & B r_{+} B r_{-}
\end{aligned}
$$

and then integrate the polar angles $\theta_{ \pm}^{*}$ asymmetrically, to finally define the $N N$-asymmetry as:

$$
\begin{aligned}
A_{L L} \equiv & -\frac{\alpha_{-} \alpha_{+}}{\sigma} \\
& \times\left(\int_{-1}^{0} d\left(\cos \theta_{-}^{*}\right)-\int_{0}^{1} d\left(\cos \theta_{-}^{*}\right)\right)\left(\int_{-1}^{0} d\left(\cos \theta_{+}^{*}\right)-\int_{0}^{1} d\left(\cos \theta_{+}^{*}\right)\right) d^{2} \sigma_{L L} \\
= & -\frac{\pi \alpha^{2} \beta}{6 s} \frac{\left(\alpha_{-} \alpha_{+}\right)}{\sigma}\left[\left(1+\beta^{2}\right)\left|F_{1}\right|^{2}+2 \operatorname{Re}\left\{F_{2}\right\}\right] B r_{+} B r_{-}
\end{aligned}
$$

\subsubsection{With Tau-direction asymmetric integration}

In order to keep the information on $F_{2}$ from the $L T$ correlation as given by Eq.(18) one must, contrary to the previous cases, integrate first in an asymmetric form the $\theta_{\tau^{-}}$angle of the outgoing Tau 


$$
\begin{aligned}
& d^{4} \sigma^{S S}(F B) \equiv\left(\int_{-1}^{0} d\left(\cos \theta_{\tau^{-}}\right)-\int_{0}^{1} d\left(\cos \theta_{\tau^{-}}\right)\right) \frac{d \sigma^{S S}\left(\vec{n}_{+}^{*}, \vec{n}_{-}^{*}\right)}{d\left(\cos \theta_{\tau^{-}}\right)} \\
& \times \frac{d \Omega_{h^{+}}}{4 \pi} \frac{d \Omega_{h^{-}}}{4 \pi} B r_{+} B r_{-} \\
&=\frac{2 \pi \alpha^{2} \beta}{3 s}\left[\left(n_{+}^{*}\right)^{x}\left(n_{-}^{*}\right)^{z}+\left(n_{+}^{*}\right)^{z}\left(n_{-}^{*}\right)^{x}\right](\mathcal{Z X}) \frac{d \Omega_{h^{+}}}{4 \pi} \frac{d \Omega_{h^{-}}}{4 \pi} B r_{+} B r_{-}
\end{aligned}
$$

with

$$
\mathcal{Z X}=\frac{1}{\gamma}\left|F_{1}\right|^{2}+\gamma\left(2-\beta^{2}\right) \operatorname{Re}\left\{F_{2}\right\}
$$

and

$$
\begin{gathered}
\left(n_{+}^{*}\right)^{x}\left(n_{-}^{*}\right)^{z}+\left(n_{+}^{*}\right)^{z}\left(n_{-}^{*}\right)^{x}=-\alpha_{+} \alpha_{-}\left(\sin \theta_{-}^{*} \cos \phi_{-} \cos \theta_{+}^{*}\right. \\
\left.+\left(\phi_{-} \leftrightarrow \phi_{+}, \theta_{-}^{*} \leftrightarrow \theta_{+}^{*}\right)\right)
\end{gathered}
$$

Then, integrating out $\theta_{-}^{*}$ and $\phi_{+}$, and performing an asymmetric integration over $\phi_{-}$, one can define a forward-backward $L T$-asymmetry as:

$$
\begin{aligned}
d \sigma_{L T} & \equiv-\left(\alpha_{-} \alpha_{+}\right) \\
& \times\left(\int_{-\pi / 2}^{\pi / 2} d \phi_{-}-\int_{\pi / 2}^{3 \pi / 2} d \phi_{-}\right)\left(\int_{0}^{2 \pi} d \phi_{+}\right)\left(\int_{-1}^{1} d\left(\cos \theta_{-}^{*}\right)\right) d^{4} \sigma^{S S}(F B) \\
& =-\frac{\pi \alpha^{2} \beta}{3 s}\left(\alpha_{-} \alpha_{+}\right)(\mathcal{Z X}) \cos \theta_{+}^{*} d\left(\cos \theta_{+}^{*}\right) B r_{+} B r_{-}
\end{aligned}
$$

Integrating now asymmetrically the $\theta_{+}^{*}$ angle, one gets the $L T$-Asymmetry

$$
\begin{aligned}
A_{L T} & \equiv\left(\int_{-1}^{0} d\left(\cos \theta_{+}^{*}\right)-\int_{0}^{1} d\left(\cos \theta_{+}^{*}\right)\right) d \sigma_{L T} \\
& =\frac{\pi \alpha^{2} \beta}{6 s} \frac{\left(\alpha_{-} \alpha_{+}\right)}{\sigma}\left[\frac{1}{\gamma}\left|F_{1}\right|^{2}+\gamma\left(2-\beta^{2}\right) \operatorname{Re}\left\{F_{2}\right\}\right] B r_{+} B r_{-}
\end{aligned}
$$

A similar procedure can be done by interchanging the angular variables of $h^{+}$by those of $h^{-}$in each of the previous steps. The result defines the $T L$ Asymmetry, which is numerically equal to the LT-Asymmetry

$$
A_{T L} \equiv A_{L T}(+\leftrightarrow-)=A_{L T}
$$




\subsection{On top of the $\Upsilon$ resonances.}

As explained in the Introduction, our aim is to measure the observables on the top of the $\Upsilon$ peak where the $\tau$ pair-production is mediated by the resonance. This has the advantage that resonant diagrams dominate the process so no contribution from box diagrams has to be considered. For Super B/Flavour factories, $\Upsilon(1 S), \Upsilon(2 S)$ and $\Upsilon(3 S)$ can be included since their decay rates into $\tau$ pairs have been measured and are sizeable.

The asymmetries obtained before are not modified when running on the top of a resonance. The differential cross section is only modified by a global $\left|H\left(M_{\Upsilon}^{2}\right)\right|^{2}$ factor, where the resonant amplitude is given by

$$
H\left(M_{\Upsilon}^{2}\right)=\frac{4 \pi \alpha Q_{b}^{2}}{M_{\Upsilon}^{2}} \frac{\left|F_{\Upsilon}\left(M_{\Upsilon}^{2}\right)\right|^{2}}{i \Gamma_{\Upsilon} M_{\Upsilon}}=-i \frac{3}{\alpha} B r\left(\Upsilon \rightarrow e^{+} e^{-}\right) .
$$

where $e-\tau$ universality has been assumed.

4.3 Contributions to the observables coming from the $Z-\gamma$ interference.

On top of the $\Upsilon$ resonance, these contributions must, in principle, also be considered. This $Z$-mediated amplitude modifies the defined asymmetries as given by:

$$
\begin{array}{ll}
A_{T T}^{Z}=-\frac{\pi \alpha^{2} \beta}{6 M_{\Upsilon}^{2}} \frac{\left(\alpha_{+} \alpha_{-}\right)}{\sigma}\left(2-\beta^{2}\right) N_{Z}, & A_{N N}^{Z}=\frac{\pi \alpha^{2} \beta}{6 M_{\Upsilon}^{2}} \frac{\left(\alpha_{+} \alpha_{-}\right)}{\sigma} \beta^{2} N_{Z}, \\
A_{L L}^{Z}=-\frac{\pi \alpha^{2} \beta}{6 M_{\Upsilon}^{2}} \frac{\left(\alpha_{+} \alpha_{-}\right)}{\sigma}\left(1+\beta^{2}\right) N_{Z}, & A_{L T}^{Z}=\frac{\pi \alpha^{2} \beta}{6 M_{\Upsilon}^{2}} \frac{\left(\alpha_{+} \alpha_{-}\right)}{\sigma} \frac{1}{\gamma} N_{Z}
\end{array}
$$

with

$$
N_{Z}=\frac{v v_{b}}{s w^{2} c w^{2}} \frac{Q_{e}}{Q_{b}} \frac{M_{\Upsilon}^{2}\left(M_{\Upsilon}^{2}-M_{z}^{2}\right)}{\left(M_{\Upsilon}^{2}-M_{z}^{2}\right)^{2}+\Gamma_{z}^{2} M_{z}^{2}}\left|H\left(M_{\Upsilon}^{2}\right)\right|^{2}
$$

and

$$
a=-\frac{1}{2}, \quad v=-\frac{1}{2}+2 s_{w}^{2} \quad v_{b}=-\frac{1}{2}+\frac{2}{3} s_{w}^{2}, \quad Q_{b}=\frac{-1}{3}, \quad Q_{e}=-1,
$$

Considering that the common numerical factor $N_{Z}$ is $(-1.567) \times 10^{-3}$, these $Z$ contributions to the asymmetries are not negligible and, in principle, have to be 
taken into account when considering the correlation observables. Fortunately, as can be seen from Eqs. (40,41), because the $\gamma-\Upsilon-Z$ interference proceeds through the vector neutral current coupling to leptons, the relevant amplitudes contributing to the asymmetries have equal structure than the ones obtained for the charge form factor $\left|F_{1}\right|^{2}$. As we will see in the following, the same combination of asymmetries which cancels the $F_{1}$ contribution will also cancel the contribution of the $\mathrm{Z}$ interference, so that it is possible to separate out $\operatorname{Re}\left\{F_{2}\right\}$ from other contributions without any ambiguities.

\section{Precision on the measurement of $\operatorname{Re}\left\{F_{2}\right\}$}

The asymmetries $T T, N N, L L$ and $L T$ (Eqs.(28), (30), (32) and (37), respectively) can be combined appropriately in order to eliminate the $\left|F_{1}\right|^{2}$ dependence. Then, the real part of the magnetic moment form factor can be obtained from the following three independent combinations of the four asymmetries:

$$
\begin{aligned}
\left(\operatorname{Re}\left\{F_{2}\right\}\right. & =\frac{\left(3-\beta^{2}\right)}{\left(\alpha_{+} \alpha_{-}\right) \beta^{2}} \times \\
& \left(\begin{array}{cccc}
\frac{4}{\gamma^{2} \beta^{2}} & 0 & 0 & \frac{4\left(2-\beta^{2}\right)}{\gamma \beta^{2}} \\
0 & \frac{4}{\left(3-\beta^{2}\right) \gamma^{2}} & 0 & \frac{4\left(1+\beta^{2}\right)}{\left(3-\beta^{2}\right) \gamma} \\
0 & 0 & -\frac{4}{\left(2-\beta^{2}\right) \gamma^{2}} & \frac{4 \beta^{2}}{\left(2-\beta^{2}\right) \gamma}
\end{array}\right) \times\left(\begin{array}{c}
A_{T T} \\
A_{L L} \\
A_{N N} \\
A_{T L}
\end{array}\right)
\end{aligned}
$$

Using these three independent combinations we can now estimate the precision that can be achieved on the determination of $\operatorname{Re}\left\{F_{2}\right\}$.

For our numerical analysis we assume the following set of integrated luminosities: Babar + Belle at $2 a b^{-1}$, and a high statistics $B$ /Flavour factory at $15 a b^{-1}$ (1 year running) and at $75 a b^{-1}$ (5 years running). The results are given in Table 1, where only the results for the $\pi^{ \pm}\left(\right.$i.e. $\left.h^{ \pm}=\pi^{ \pm}\right)$decay channel, for the traced $\tau^{ \pm}$, are quoted. Results for the $\rho^{ \pm}\left(h^{ \pm}=\rho^{ \pm}\right)$and $\rho^{ \pm}-\pi^{\mp}$ $\left(h^{ \pm}=\rho^{ \pm}, h^{\mp}=\pi^{\mp}\right)$ channels are 4 and 2 times looser, respectively, than those for the $\pi^{ \pm}$ones. They poorly contribute to increase the precision measurement of $\operatorname{Re}\left\{F_{2}\right\}$ but we have taken them into account, for completeness, in the global result given in Table 1. This global result has been obtained by

considering all correlation channels of Eq.(44), for all possible combination of 
the Tau decays to $\pi$ 's and $\rho$ 's. For all the results presented here only statistical errors are considered. The comparison with the results given in Ref. [8] shows that observables obtained with polarized electrons are better by a factor 3 than those given for unpolarized electron-beams. In addition, one must be aware that experimental uncertainties may be bigger for the detection in coincidence of two Taus, in order to get correlations, than for single Tau polarization, so that bounds from correlations may be looser that those for single Tau observables.

Table 1

Sensitivity of the $F_{2}\left(q^{2}\right)$ form factor measurement at the $\Upsilon$ energy

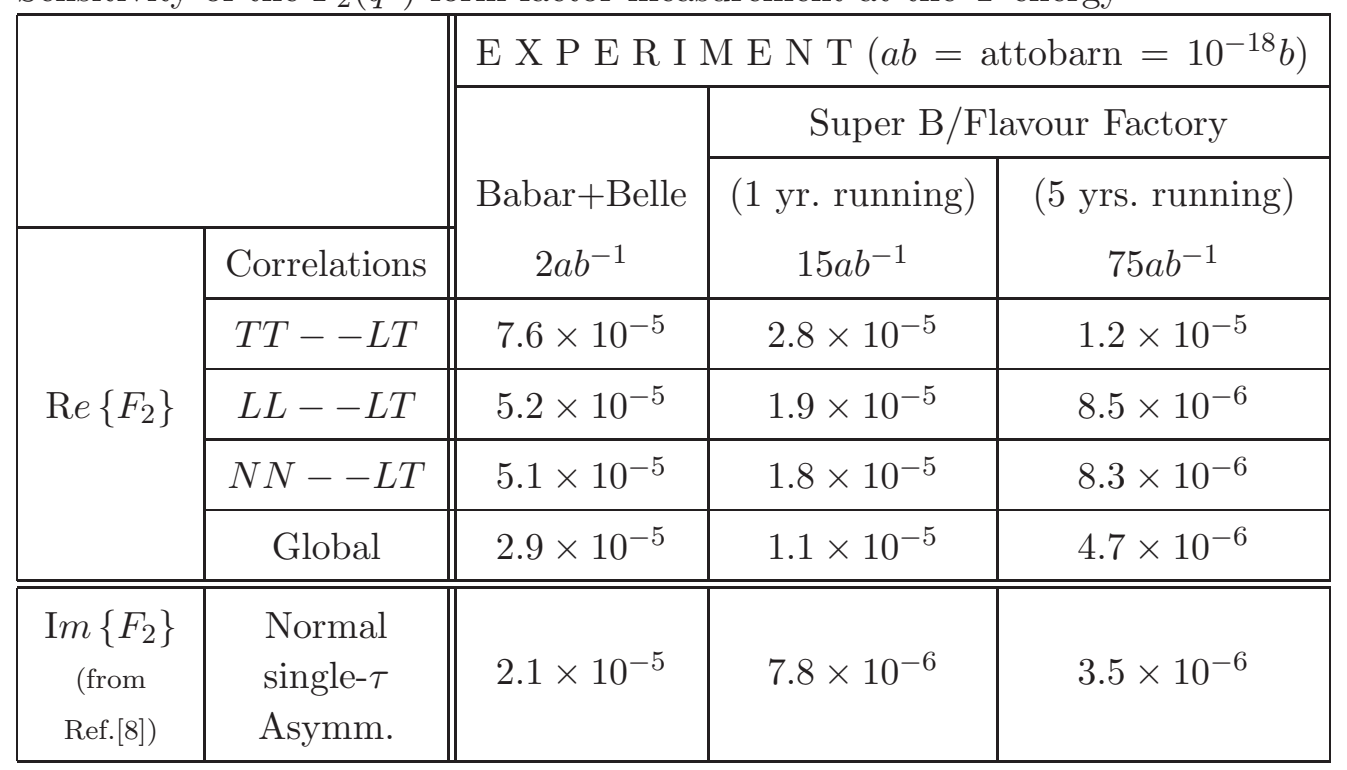

\section{Conclusions}

We have estimated the precision that can be achieved in the determination of the $Q E D$ scale and flavour effects in the measurement of the $F_{2}$ magnetic moment form factor, at $\Upsilon$ energies, for unpolarized $e^{+} e^{-}$collisions. In Ref. [8] we already showed that the imaginary part of $F_{2}$ can be determined from the Normal Asymmetry of the decay products for a normal polarization of a single Tau. In this paper, we have shown that, for unpolarized electron beams, the real part of the form factor needs the measurement of correlations on the Tau decay products of both polarized Taus. We have defined correlations sensitive to the $F_{2}$ form factor and found three independent combinations of them to get its value without any ambiguities, eliminating the contribution of the charge form factor $F_{1}$ and the $Z-\gamma$ interference. Combining all channels, the sensitivity that can be achieved is of the order of $10^{-5}-10^{-6}$, which allows the measurement of the magnetic moment form factor $F_{2}\left(M_{\Upsilon}^{2}\right)$, given in Eq.(8), 
up to a precision of a few per cent. This result shows that Super B/Flavour factories can bring, for the first time, important information on the rather poorly known magnetic properties of the Tau.

\section{Acknowledgements}

This work has been supported by the Ministerio de Educación y Ciencia (MEC) and FEDER, under the grants FPA2005-00711 and FPA2005-01678, and by Pedeciba and DINACYT-PDT-54/94-Uruguay.

\section{References}

[1] J. Abdallah et al. [DELPHI Collaboration], Eur. Phys. J. C 35 (2004) 159.

[2] F. Cornet and J. I. Illana, Phys. Rev. D 53 (1996) 1181.

[3] W.-M. Yao et al., Journal of Physics G 33, (2006) 1.

[4] G.A. González-Sprinberg, A. Santamaria, J. Vidal, Nucl. Phys. B 582 (2000) 3 .

[5] M. A. Samuel, G. w. Li and R. Mendel, Phys. Rev. Lett. 67 (1991) 668;

M. A. Samuel, G. w. Li and R. Mendel, Phys. Rev. Lett. 69 (1992) 995. Erratum;

S. Eidelman and M. Passera, Mod. Phys. Lett. A 22 (2007) 159.

[6] J. Schwinger, Phys. Rev. 73 (1948) 416, Phys. Rev. 76 (1949) 790.

[7] SuperB Conceptual Design Report, http:www.pi.infn.it/SuperB .

[8] J. Bernabéu, G.A. González-Sprinberg, J. Papavassiliou and J. Vidal, Nucl. Phys. B 790 (2008) 160.

[9] J. Bernabéu, G. A. González-Sprinberg and J. Vidal, Nucl. Phys. B 701 (2004) 87.

[10] J. Bernabéu, G.A. González-Sprinberg and J. Vidal, Nucl. Phys. B 763 (2007) 283.

[11] J. H. Kuhn, Phys. Lett. B 313 (1993) 458.

[12] Y.S. Tsai Phys. Rev. D 4 (1971) 2821. 